\title{
LA COMUNICACIÓN ENTRE PROFESORES Y ESTUDIANTES DE PERIODISMO: CANALES Y ESPACIOS DE INTERACCIÓN EN ENTORNOS ON LINE DE APRENDIZAJE
}

Celia Berná-Sicilia1: Universidad Católica San Antonio. España cberna@pdi.ucam.edu

Carolina Fernández-Castrillo: Universidad a Distancia de Madrid. España c.fernandez.c@udima.es

\section{RESUMEN}

La rápida y eficaz implantación de las Tecnologías de la Información y de la Comunicación (TIC) al ámbito de educación superior ha propiciado la instauración de prácticas innovadoras y la creación de canales de transmisión del conocimiento alternativos a los mecanismos tradicionales, con objeto de permitir la adquisición de las competencias exigidas en la vida profesional y de responder a las necesidades que plantea la sociedad actual. Desde el Grado de Periodismo de la Universidad a Distancia de Madrid (UDIMA) se ha instaurado un nuevo modelo de acción formativa, en la línea de los criterios establecidos por el Espacio Europeo de Educación Superior (EEES), basado en nuevas estrategias de interacción con el alumnado. Este estudio ofrece algunas claves para mejorar la eficacia comunicativa de las nuevas estrategias docentes en el ámbito del e-Learning. Al mismo tiempo, se pretende evaluar el nivel de implantación de las TIC en este grado, así como el nivel de satisfacción de los estudiantes y los profesores con respecto al modo en que se desenvuelve la comunicación durante el proceso formativo.

PALABRAS CLAVE: Comunicación multimedia - e-Learning - innovación docente Periodismo - Tecnologías de la Información y la Comunicación (TIC

\footnotetext{
${ }^{1}$ Autor correspondiente

Celia Berná-Sicilia: Universidad Católica San Antonio. Murcia. España

Correo: cberna@pdi.ucam.edu
} 


\title{
COMMUNICATION BETWEEN TEACHERS AND STUDENTS OF JOURNALISM: INTERACTION SPACES IN E-LEARNIG ENVIRONMENTS
}

\begin{abstract}
The fast implementation and effective introduction of Information Technology (IT) in Higher Education has stimulated innovative practices and the creation of alternative knowledge tools in contraposition to traditional learning systems. This new situation guarantees the acquisition of new skills required both in professional life and in current society. The Journalism Degree at Madrid Open University (UDIMA) has introduced a new educational model, in line with the criteria established by the European Higher Education Area (EHEA) based on new strategies of interaction with students. This study provides some clues to improve the communication effectiveness of new teaching strategies in e-Learning. At the same time, we will assess the level of implementation of IT in Journalism Degree, as well as students and teachers's satisfaction with new communicative processes during the training process.
\end{abstract}

KEY WORDS: e-Learning - Information Technology (IT) - Multimedia Communication - Journalism - Teaching Innovation.

\section{INTRODUCCIÓN}

La incorporación de las nuevas tecnologías de la información y de la comunicación (TICs) en el diseño instruccional universitario está propiciando la aparición de nuevos canales para la difusión de contenidos formativos.

Esta nueva realidad exige reformular la relación entre los principales agentes del sistema educativo, máxime en el Grado de Periodismo para fomentar el desarrollo de las habilidades comunicativas. Asimismo, en el ámbito del e-Learning, el establecimiento de una relación fluida entre el profesor y sus alumnos resulta determinante para la consecución de los objetivos docentes.

Conscientes de la necesidad de adaptar el sistema formativo al nuevo contexto tecnológico, el profesorado de la Universidad a Distancia de Madrid (UDIMA) ha optado por instaurar un nuevo modelo de acción formativa. Las nuevas estrategias metodológicas y de interacción con el alumnado han sido diseñadas ad hoc para 
En efecto, el nuevo panorama socio-comunicativo está íntimamente vinculado a las nuevas tecnologías, al igual que los sistemas de e-Learning, en los que resulta indispensable facilitar el desarrollo de actividades formativas en las que predomine una comunicación próxima y en tiempo real, posibilitando así la creación de contenidos hipertextuales y multimedia (texto, sonido, música, imagen fija, vídeo, etc.), favoreciendo la realización de trabajos en grupo y la formulación de un amplio abanico de actividades de autoevaluación con objeto de que el estudiante logre adquirir las competencias que plantea el mundo actual.

Los nuevos desafíos tecnológicos y comunicativos que se exigen en la actual vida profesional afectan de modo particular al ámbito periodístico. Por este motivo, junto a la consecución de los objetivos docentes, se pretende que los futuros comunicadores logren afianzar sus conocimientos sobre las nuevas herramientas on- line $\mathrm{y}$, al mismo tiempo, disfruten del proceso de aprendizaje, gozando de un contacto directo con el profesorado.

La acción comunicativa estratégica llevada a cabo en esta universidad trata de salvar las eventuales carencias que tradicionalmente se vinculan a los sistemas educativos a distancia que pueden conducir a la deserción por parte de los estudiantes - pues redunda en la motivación y en la activación de un aprendizaje significativo adaptado a las necesidades de la sociedad actual - y, al mismo tiempo, viene a demostrar que se pueden llegar a superar los resultados obtenidos en la enseñanza presencial.

A continuación, a partir de los resultados obtenidos en el empleo de las TICs en el diseño y desarrollo de actividades formativas del Grado de Periodismo en la UDIMA, se ofrecerán algunas claves para la mejora en la interacción con el alumnado con el objeto de crear espacios comunicativos ágiles y fluidos que transmitan una sensación de proximidad.

\section{METODOLOGÍA}

La presente investigación se basa en el análisis de las anteriores escuelas de pensamiento que han tratado el tema que nos ocupa. Se trata de analizar los principales medios existentes hoy y aplicarles un método analítico textual que permita producir inferencias fenomenológicas o estructuras de pensamiento novedoso basado en este tipo de reflexiones que crean un puente entre las ideas anteriores (y sus realidades descritas) y las más modernas. 


\section{ANÁLISIS Y DISCUSIÓN}

\subsection{El Grado de Periodismo en el EEES}

En la actualidad, la apuesta por la incorporación de las nuevas tecnologías al ámbito educativo constituye un objetivo común en el marco del Espacio Europeo de Educación Superior (EEES). Dentro de esta línea de actuación, en España las nuevas medidas adoptadas tras la adaptación al Plan Bolonia pretenden "dotar a los alumnos de unas competencias que les permitan seguir aprendiendo y encontrar por sí mismos los caminos del conocimiento y la resolución de problemas" (Sierra y Cabezuelo, 2009, p.2). De ahí la importancia de la aplicación de las nuevas tecnologías en esta nueva concepción del aprendizaje.

En línea con este planteamiento, el Grado de Periodismo de la Universidad a Distancia de Madrid surge de la necesidad de satisfacer la demanda de profesionales formados en la profundidad teórica, el deber ético, el estado del arte tecnológico y las habilidades prácticas por parte de las empresas e instituciones del ámbito de la comunicación periodística, así como las de la sociedad a la que están llamados a informar los periodistas del futuro.

Dentro de los objetivos y las competencias curriculares marcadas por el EEES, cabe destacar que son ya muchas y provechosas las experiencias docentes que se están desarrollando en el marco de las asignaturas de este grado, desde la convicción de que la tecnología constituye una garantía de excelencia, siempre y cuando responda a los objetivos educativos y profesionales perseguidos.

En la interacción con los estudiantes y en el desarrollo de la labor formativa, la UDIMA favorece la creación de un discurso multimedia, tanto desde dentro del propio entorno educativo -las aulas virtuales- como desde fuera de él -Elluminate, Second Life, Google docs, Google sites, etc.-.

El despliegue de una amplia gama de códigos, estrategias y herramientas permite alcanzar una comunicación fluida y eficaz en la práctica docente capaz de crear espacios de trabajo colaborativos que fomenten la motivación de los alumnos y la instauración de un nuevo modelo de aprendizaje.

\subsection{La acción comunicativa estratégica en el Grado de Periodismo de la UDIMA}

El objetivo de alcanzar el éxito comunicativo pone en marcha un complejo engranaje 
Los procesos de teleformación se desenvuelven bajo la influencia de factores diversossoledad del alumno, formación previa, ausencia de comunicación directa, etc.- Esta singularidad imprime ciertas peculiaridades que hacen que la comunicación constituya uno de los pilares básicos sobre los que se asienta el éxito de la formación y el seguimiento de la evaluación continua y, a la vez, representa un reto importante al que deben enfrentarse los agentes universitarios para salvar la distancia física que existe entre ellos. La acción comunicativa estratégica en el Grado de Periodismo en particular y en la UDIMA en general se fundamenta en tres aspectos esenciales:

a) Las técnicas desplegadas: tácticas y prácticas habituales aplicadas por el profesor-tutor para favorecer la tarea formativa y para evitar el abandono.

b) Las herramientas utilizadas: dispositivos tecnológicos facilitadores de la transmisión de contenidos.

c) El uso de multiplicidad de códigos: mezcla de distintos sistemas de comunicación para ampliar las posibilidades expresivas.

La combinación simultánea de estos tres mecanismos se orienta hacia el aprendizaje efectivo en el contexto de la enseñanza del Grado de Periodismo, imprimiendo rasgos específicos a los intercambios que se producen entre aquellos que participan en el proceso formativo, sobre todo frente a los sistemas tradicionales.

\section{Técnicas}

La tarea formativa en el Grado de Periodismo se desenvuelve gracias a la articulación de algunas prácticas orientadas a evitar el abandono y a favorecer el desarrollo y la consecución exitosa de la comunicación profesor-alumno. De entre las estrategias y prácticas comunicativas más habituales podemos señalar algunas que destacan por su efectividad:

a) Sistema de evaluación continua: seguimiento intensivo del aprendizaje del alumno a través de distintos tipos de actividades (cuestionarios, tareas, WebQuests, etc.). De este modo, siguiendo las directrices de Bolonia, la comunicación con el alumno es constante durante todo el proceso, pues la aprehensión de los contenidos se efectúa de manera progresiva y el estudiante puede afrontar el examen con mayores garantías de éxito.

b) Cronograma que detalla la secuenciación de actividades durante el curso y el tiempo estimado de realización de cada una de ellas.

c) Instrucciones detalladas para efectuar las actividades: PDFS, tutoriales, vídeos, tutorías personalizadas, etc.

d) Inmediatez en el envío de respuestas y correcciones. 
f) Tablón de anuncios: mensajes de refuerzo y de recuerdo que el profesor periódicamente va enviando al grupo que le ha sido asignado en cada asignatura.

\section{Herramientas}

La UDIMA ha optado por realizar una apuesta decidida por la tecnología desde la convicción de que su empleo en la impartición de la enseñanza superior se adecua a los requisitos que impone la reforma de Bolonia y constituye una auténtica garantía de futuro.

En el proceso formativo del Grado de Periodismo de la UDIMA se despliega una amplia variedad de dispositivos tecnológicos, a pesar de que, como veremos, algunas herramientas parecen ser prioritariamente empleadas por los docentes en el desarrollo de su labor formativa.

En este marco institucional, la docencia se desarrolla fundamentalmente a través de la plataforma de teleformación Moodle. Este entorno virtual constituye el sistema de gestión de aprendizaje más extendido en el ámbito educativo.

Con todo, podemos distinguir, dos grandes grupos de herramientas, dentro de los dispositivos que facilitan el desarrollo de la comunicación en las tareas docentes de esta universidad:

a) Herramientas integradas en el Aula Virtual de la plataforma Moodle:herramientas internas.

b) Herramientas que se sitúan fuera del Aula Virtual de la plataforma Moodle: herramientas externas. 
Tabla 1. Herramientas comunicativas internas y externas en el grado de Periodismo. Fuente: Elaboración propia.

\begin{tabular}{|c|c|}
\hline Herramientas internas & Herramientas externas \\
\hline $\begin{array}{l}\text { Foros: (de discusión y de tutorías, } \\
\text { fundamentalmente): sistema de } \\
\text { mensajería } \\
\text { para el intercambio de opiniones } \\
\text { sobre un tema concreto o para la } \\
\text { resolución de dudas. }\end{array}$ & $\begin{array}{l}\text { Elluminate: software educativo que } \\
\text { permite al } \\
\text { docente proyectar presentaciones, con la } \\
\text { ayuda de } \\
\text { recursos de vídeo, audio, pizarra virtual o chat. } \\
\text { Además, este dispositivo ofrece algunas otras } \\
\text { posibilidades, como la de grabar la sesión para } \\
\text { que aquellos alumnos que no han podido } \\
\text { asistir a la sesión la puedan ver en cualquier } \\
\text { momento }^{2} \text {. }\end{array}$ \\
\hline $\begin{array}{l}\text { Chat: sistema de mensajería } \\
\text { instantánea que permite la interacción } \\
\text { en tiempo real. }\end{array}$ & $\begin{array}{l}\text { Second Life (SL): metaverso desarrollado } \\
\text { por } \\
\text { Linden Lab. Se trata de un entorno virtual } \\
\text { tridimensional, accesible de forma gratuita } \\
\text { desde } \\
\text { Internet, que permite a los usuarios } \\
\text { interactuar entre ellos por medio de avatares } \\
\text { gracias al uso de múltiples programas de } \\
\text { interface (viewers) }{ }^{3} \text {. }\end{array}$ \\
\hline $\begin{array}{l}\text { Consultas: herramienta que permite } \\
\text { recopilar } \\
\text { Información sobre uno o varios temas } \\
\text { concretos. }\end{array}$ & $\begin{array}{l}\text { Youtube: es un sitio web en que el los } \\
\text { usuarios pueden subir y compartir vídeos. } \\
\text { Los enlaces a vídeos de Youtube pueden ser } \\
\text { incorporados en las aulas virtuales incrustando } \\
\text { el código HTML }{ }^{4} \text {. }\end{array}$ \\
\hline
\end{tabular}

\footnotetext{
${ }^{2}$ Asimismo, esta herramienta favorece la existencia de una mayor interacción en la relación profesorestudiante: los alumnos pueden hablar, escribir en la pizarra, levantar la mano, aplaudir o mostrar desacuerdo a través de un simpático icono de silbido, etc.

${ }^{3}$ Desde el ámbito educativo se recurre a SL principalmente para promover la participación de actividades individuales o en grupo y la asistencia a clases virtuales. UDIMA cuenta con una sede virtual

en Second

Life.

$\mathrm{Su}$

URL 


\begin{tabular}{|c|c|}
\hline $\begin{array}{l}\text { Tareas: dispositivo para el envío de } \\
\text { actividades por parte de los } \\
\text { alumnos y su correspondiente } \\
\text { evaluación. }\end{array}$ & $\begin{array}{l}\text { Redes sociales: las principales redes } \\
\text { sociales en el } \\
\text { ámbito educativo son Facebook y Twitter. La } \\
\text { primera es un sitio web abierto en el } \\
\text { que predomina una comunicación } \\
\text { multimedia. Sin embargo, la estructura de } \\
\text { Twitter se basa en el microblogging. }\end{array}$ \\
\hline $\begin{array}{l}\text { Glosarios: diccionario virtual que } \\
\text { facilita la } \\
\text { creación de entradas y sus } \\
\text { correspondientes definiciones para su } \\
\text { consulta. }\end{array}$ & $\begin{array}{l}\text { Google: las aplicaciones Google docs y } \\
\text { Google sites } \\
\text { posibilitan el intercambio de conocimiento y } \\
\text { la realización de tareas colaborativas y } \\
\text { grupales en línea }{ }^{5} \text {. }\end{array}$ \\
\hline $\begin{array}{l}\text { Wikis: mecanismo que permite } \\
\text { desarrollar contenidos de una } \\
\text { asignatura de manera colaborativa. }\end{array}$ & $\begin{array}{l}\text { Correo electrónico: favorece un trato } \\
\text { personalizado y ofrece respuestas a consultas } \\
\text { de los estudiantes de manera prácticamente } \\
\text { inmediata. }\end{array}$ \\
\hline $\begin{array}{l}\text { Calendario: almanaque virtual que } \\
\text { informa de los eventos que tienen } \\
\text { lugar durante el curso. Esta } \\
\text { herramienta facilita la optimización en } \\
\text { la gestión y en la organización del } \\
\text { tiempo. }\end{array}$ & $\begin{array}{l}\text { Teléfono: especialmente indicado para la } \\
\text { formulación de cuestiones concretas y para } \\
\text { obtener un trato más cercano. Cada } \\
\text { asignatura cuenta con un servicio de tutorías } \\
\text { telefónicas. }\end{array}$ \\
\hline
\end{tabular}

A continuación, se ofrecen los datos sobre cómo evalúan exactamente los docentes de la UDIMA estas herramientas comunicativas, atendiendo fundamentalmente a dos parámetros:

a) Nivel de utilización.

b) Nivel de satisfacción.

La información ha sido recopilada a partir de una encuesta rellenada por los 15 profesores que imparten docencia en el Grado de Periodismo en la UDIMA: 
La valoración se ha hecho según una escala Likert del 1 al 5 . El 5 constituye el valor asociado a un mayor nivel de utilización o de satisfacción, mientras que el 1 se vincula con el menor nivel de utilización o de satisfacción.

De acuerdo con el primer criterio, los dispositivos más utilizados son el correo electrónico, las tareas, los foros, los documentos en PDF o Power Point, el teléfono y Elluminate.

En consonancia con los datos disponibles, estas herramientas se incorporan de manera habitual y cotidiana en el quehacer profesional de los docentes de la UDIMA para el desarrollo de su labor formativa.

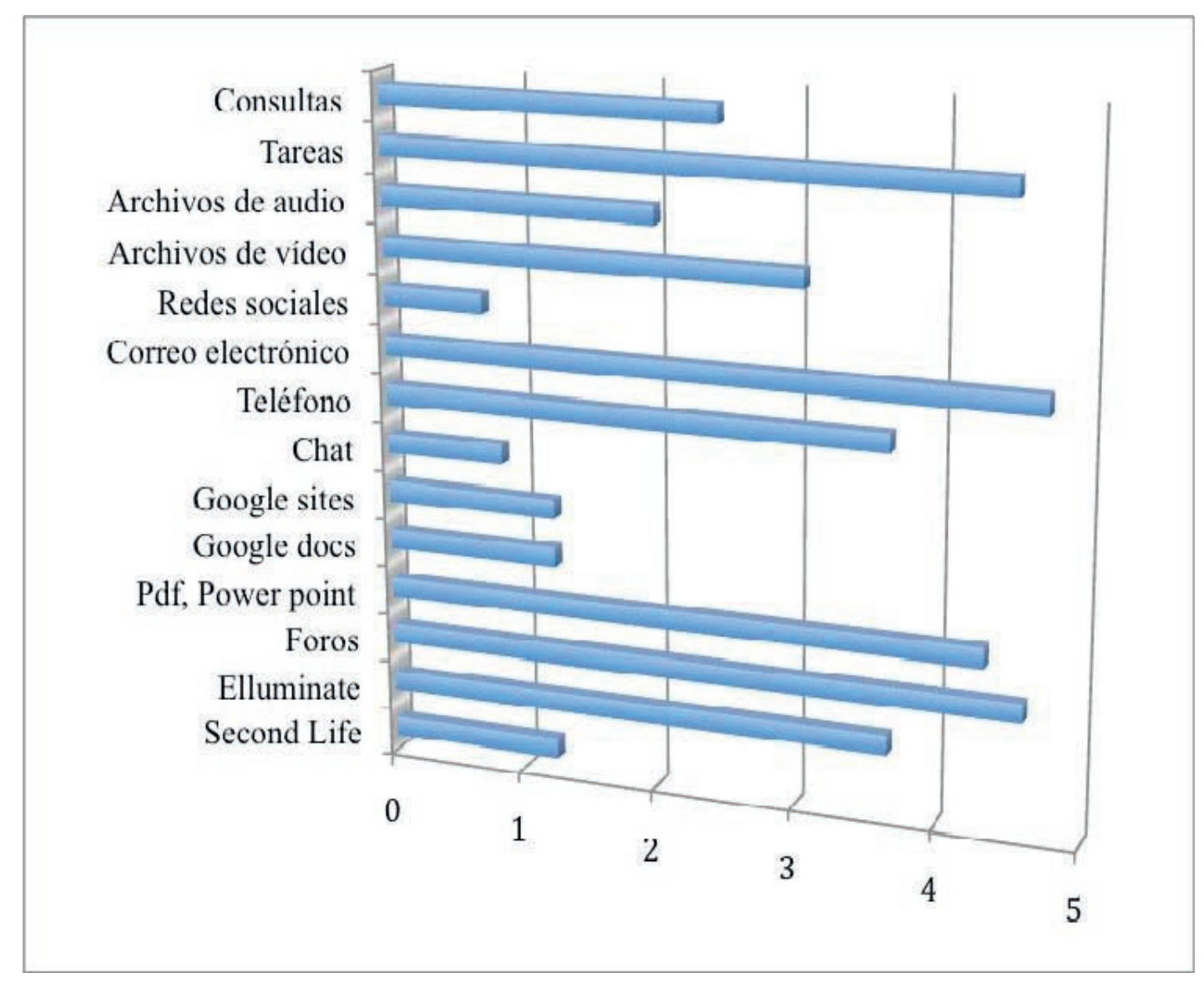

Figura 1. Nivel de utilización de las herramientas comunicativas en el Grado de Periodismo de la UDIMA. Fuente: Elaboración propia. 
Según el segundo criterio, que se vincula al nivel de satisfacción de los profesores con respecto a las herramientas, teniendo en cuenta su eficacia comunicativa, los dispositivos mejor valorados se corresponden con las tareas, el teléfono y los documentos Power Point y PDF, si bien se encuentran seguidos muy de cerca por otros recursos como Elluminate, los foros y el correo electrónico.

A la luz de estos datos, parece relevante destacar que las herramientas más utilizadas figuran también entre las mejor valoradas por los profesores por adecuarse mejor a los objetivos docentes perseguidos.

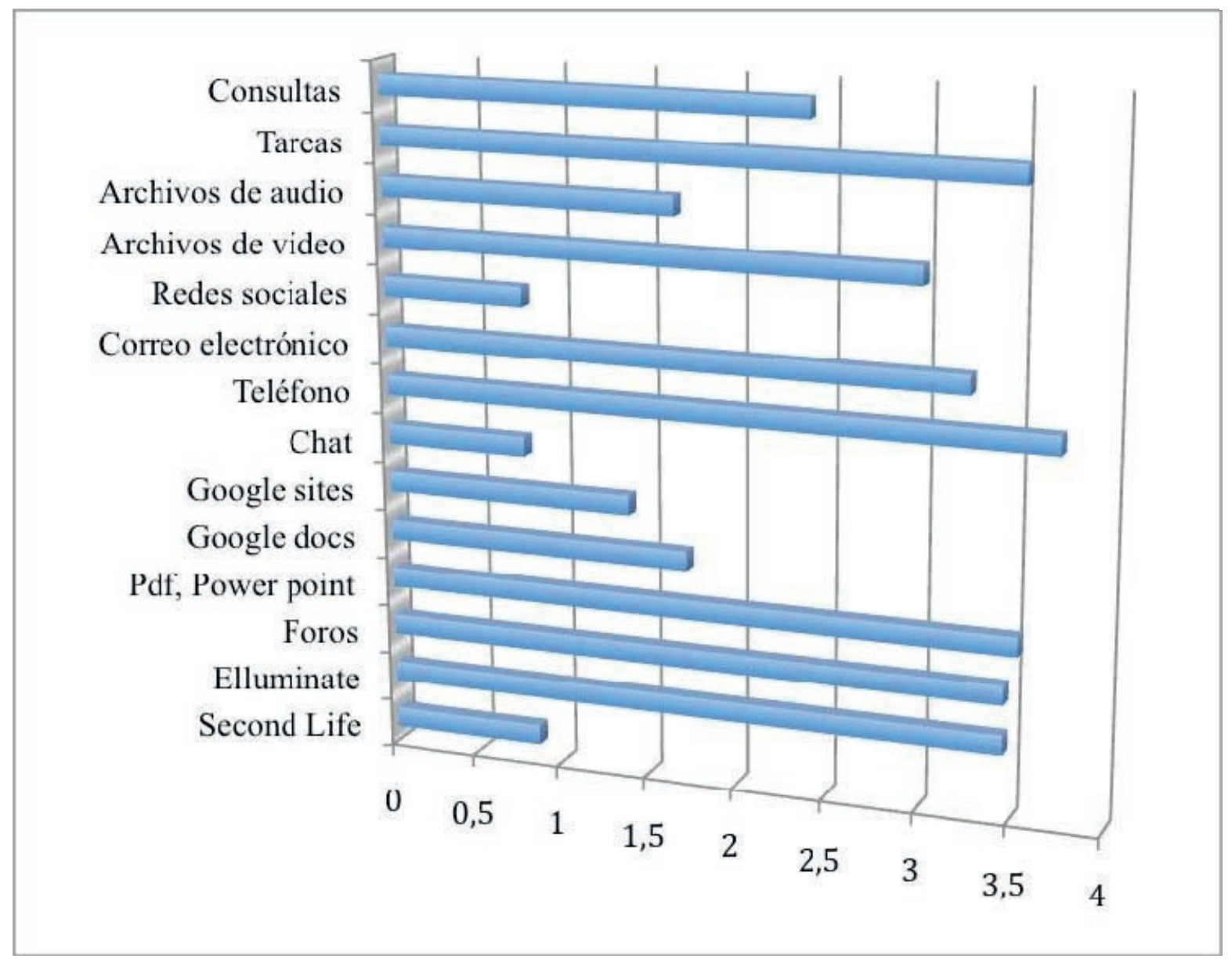

Figura 2. Nivel de satisfacción de los docentes con respecto a las herramientas comunicativas en el Grado de Periodismo de la UDIMA. Fuente: Elaboración propia

Finalmente, se proporcionan los resultados generales de satisfacción de los alumnos con respecto a algunas de las herramientas más importantes y en relación con algunos

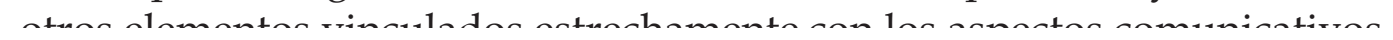


Los datos han sido extraídos de la encuesta de satisfacción general que se pasa a los estudiantes al finalizar el primer semestre del curso académico 2010-2011 (periodo de octubre a febrero). Al igual que en el caso anterior, la valoración se efectúa en una escala Likert de 1 a 5. Además, ofrecemos también los datos generales de la UDIMA para poder establecer un contraste.

Tabla 2. Valoración de las herramientas comunicativas por parte de los alumnos.

Fuente: Elaboración propia.

\begin{tabular}{|c|c|c|}
\hline Ítems & $\begin{array}{l}\text { Media en el Grado de } \\
\text { Periodismo }\end{array}$ & $\begin{array}{l}\text { Media en } \\
\text { UDIMA }\end{array}$ \\
\hline $\begin{array}{l}\text { Información sobre la Guía Docente de } \\
\text { las asignaturas }\end{array}$ & 4,53 & 4,41 \\
\hline $\begin{array}{l}\text { Información sobre el sistema de } \\
\text { evaluación }\end{array}$ & 4,51 & 4,46 \\
\hline $\begin{array}{l}\text { La información proporcionada por el } \\
\text { profesor me ha permitido planificar mi } \\
\text { aprendizaje }\end{array}$ & 4,53 & 4,35 \\
\hline $\begin{array}{l}\text { Explicación clara de dudas y conceptos por } \\
\text { parte del profesor }\end{array}$ & 4,53 & 4,48 \\
\hline Organización del Aula & 4,53 & 4,43 \\
\hline Tareas & 4,66 & 4,64 \\
\hline Glosarios & 3,72 & 3,89 \\
\hline Foros & 4,48 & 4,37 \\
\hline Chat & 3,02 & 2,95 \\
\hline Consultas & 4,36 & 4,29 \\
\hline Elluminate & 4,2 & 4,16 \\
\hline Second Life & 2,67 & 2,82 \\
\hline Google docs & 3,58 & 3,33 \\
\hline Google sites & 3,53 & 2,95 \\
\hline Tutorías telefónicas & 3,5 & 3,36 \\
\hline
\end{tabular}


En virtud de la información disponible, podemos establecer que los estudiantes valoran positiva o muy positivamente las herramientas y los recursos comunicativos empleados en la formación de grado. Además, tal como podemos observar, la media del Grado de Periodismo resulta levemente superior a la media general de la UDIMA en casi todos los ítems evaluados.

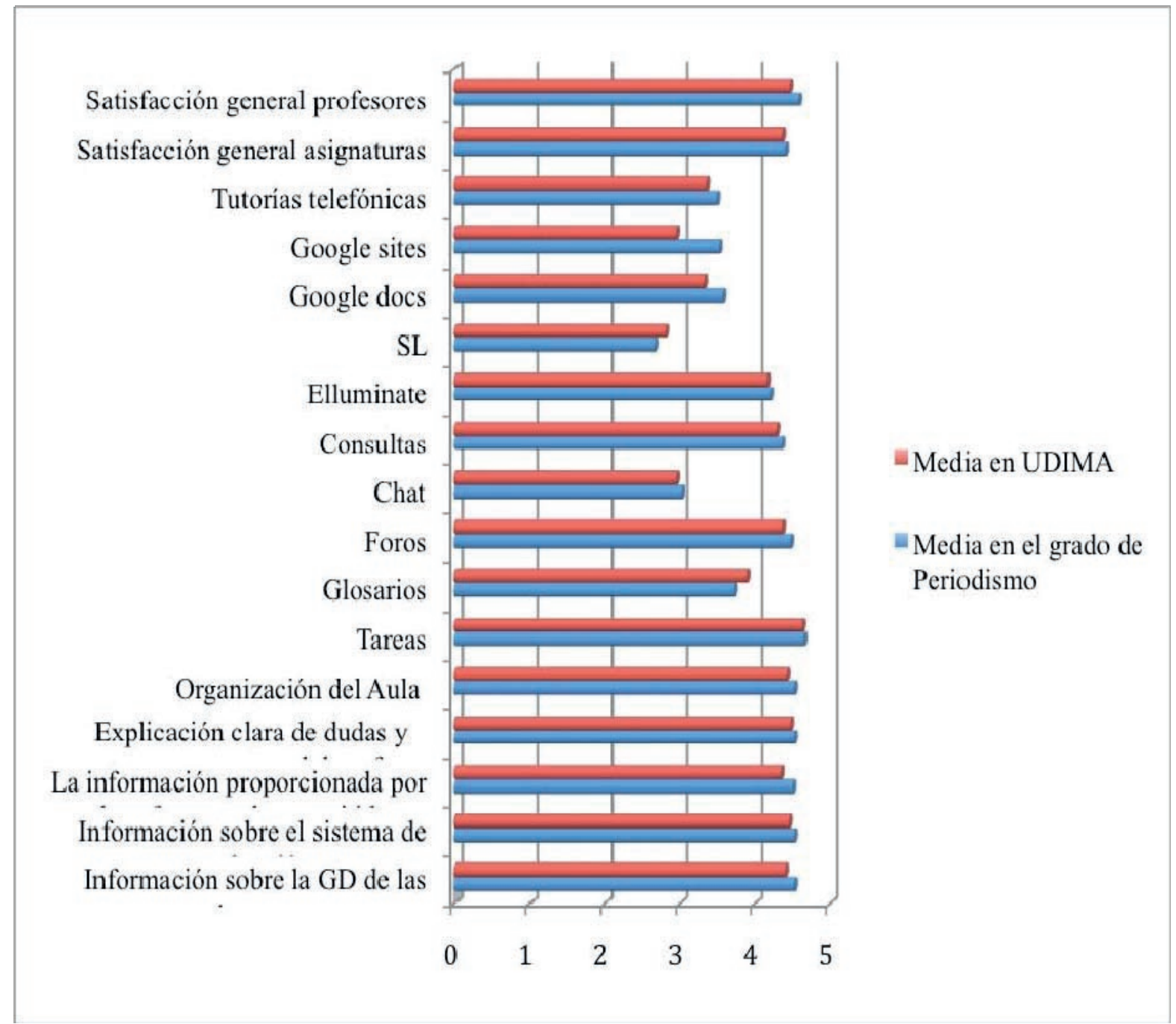

Figura 3. Nivel de satisfacción de los estudiantes con respecto a las herramientas y recursos comunicativos de la UDIMA. Fuente: Elaboración propia.

\section{Multiplicidad de códigos}

La combinación de diferentes tipos de técnicas y de distintos dispositivos tecnológicos facilita la activación de un lenguaje multimedia dentro del proceso de enseñanza-

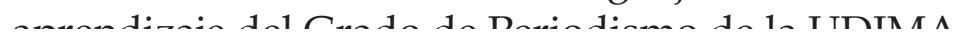


a) Código lingüístico (series visual y acústica lingüísticas): elementos verbales propiamente dichos (orales $y$ escritos, aunque fundamentalmente escritos).

b) Código paralingüístico (serie visual paralingüística): diagramación, ubicación de los elementos dentro del Aula, tipografía, etc.

c) Código icónico (serie visual no lingüística): imágenes, gráficos y vídeos.

En este tipo de código también quedarían incluidos los símbolos e iconos asociados a cada uno de los tipos de recurso del Aula ( $>$ foro, >tarea) y los emoticonos ocasionalmente utilizados en la comunicación con los estudiantes para la correcta desambiguación de mensajes.

Cada código utiliza signos de distinta naturaleza, de modo que, en general, podemos hablar de la existencia de una suerte de lenguaje mixto y plural en el marco de la comunicación profesor-alumno que surge a partir de la concurrencia de distintos sistemas comunicativos.

Con todo, es preciso advertir que, aunque se utilizan distintos códigos de manera combinada, en general, en virtud de las especificidades que caracterizan el contexto de la educación a distancia, la comunicación visual manifiesta un claro predominio en los intercambios de información del Grado de Periodismo de la UDIMA.

Veamos, a continuación, un cuadro resumen que evidencia la mezcla de códigos que se produce en los procesos comunicativos que se desarrollan en el Grado de Periodismo. 
Tabla 3. Códigos empleados en la comunicación con el alumno en el Grado de Periodismo de la Udima. Fuente: Elaboración propia.

\begin{tabular}{|c|c|c|}
\hline Series & En Moodle & Fuera de Moodle \\
\hline $\begin{array}{l}\text { Serie visual lingüística: } \\
\text { código lingüístico }\end{array}$ & $\begin{array}{lr}\text { Chat, } & \text { PDFS, } \\
\text { presentaciones en } & \text { Power } \\
\text { Point, recursos } & \text { web, } \\
\text { consultas, foros, tareas, } \\
\text { glosarios, wikis. }\end{array}$ & $\begin{array}{l}\text { Correo electrónico, redes } \\
\text { sociales, Google docs, } \\
\text { Google sites }\end{array}$ \\
\hline $\begin{array}{l}\text { Serie visual } \\
\text { paralingüística: } \\
\text { código paralingüístico }\end{array}$ & $\begin{array}{l}\text { Calendario, ordenación de } \\
\text { contenidos en el Aula por } \\
\text { semanas, etiquetas, uso } \\
\text { de negrita, etc. }\end{array}$ & - \\
\hline $\begin{array}{l}\text { Serie visual no lingüística: } \\
\text { código icónico }\end{array}$ & $\begin{array}{l}\text { Iconos de } \text { Moodle para } \\
\text { cada tipo de actividad, } \\
\text { emoticonos en mensajes, } \\
\text { etc. }\end{array}$ & $\begin{array}{l}\text { Second Life, redes sociales, } \\
\text { google sites, vídeos. }\end{array}$ \\
\hline $\begin{array}{l}\text { Serie acústica lingüística: } \\
\text { código lingüístico }\end{array}$ & $\begin{array}{l}\text { Archivos de voz, tutorías } \\
\text { telefónicas, etc. }\end{array}$ & $\begin{array}{l}\text { Teléfono, Elluminate, } \\
\text { Second } \\
\text { Life, archivos de audio. }\end{array}$ \\
\hline
\end{tabular}

\section{CONCLUSIONES}

\subsection{Recapitulación}

La propuesta formativa de la UDIMA pone en marcha una acción comunicativa estratégica que se asienta, como hemos señalado, sobre canales y espacios de interacción diversos. Este sistema facilita la adquisición de las competencias por parte de los estudiantes de acuerdo con las exigencias que marca la práctica profesional periodística en la actualidad y permite, asimismo, obtener resultados a veces incluso más satisfactorios y provechosos que los conseguidos en la enseñanza presencial.

Este breve análisis proporciona una visión detallada de los mecanismos que el profesorado del Grado de Periodismo emplea a la hora de activar el flujo 
Las líneas y directrices que marca Bolonia en los renovados planes de estudio universitarios y los rasgos singulares que caracterizan los contextos de e-learning imponen nuevos rumbos en el desarrollo y perfeccionamiento de los procesos comunicativos para facilitar la tarea formativa.

La optimización de la comunicación profesor-alumno se logra en la UDIMA gracias al desarrollo de una compleja acción estratégica que pivota sobre tres mecanismos esenciales activados simultáneamente durante el ejercicio de la práctica docente: técnicas, herramientas y multiplicidad de códigos.

Nuestro estudio revela que los docentes del Grado de Periodismo utilizan una amplia variedad de códigos desarrollando lenguaje multimediático, aplican técnicas de refuerzo y de seguimiento de las actividades planteadas durante el curso y emplean, a su vez, herramientas tecnológicas diversas, buscando en todo momento la eficacia comunicativa en el desempeño de su labor formativa.

A pesar de que no existe total homogeneidad por cuanto respecta al grado de implantación y desarrollo de las técnicas, de las herramientas y del lenguaje multimedia en las distintas asignaturas del grado de Periodismo, en virtud de la información de que disponemos, las estrategias puestas en marcha para favorecer los intercambios de información y el amplio bastidor de recursos -externos e internosusados en la actividad docente de esta universidad propician el establecimiento de una comunicación fluida entre los distintos agentes universitarios que facilita la progresión y el éxito del proceso de enseñanza-aprendizaje.

Por otra parte, la utilización de un código híbrido que entremezcla distintos tipos de lenguajes permite multiplicar las posibilidades expresivas $\mathrm{y}$, además, ayuda a acercar el discurso docente universitario a los tipos discursivos multimedia propios del siglo XXI.

Habida cuenta de los resultados que arrojan las encuestas de satisfacción completadas tanto por los estudiantes como por los profesores de la UDIMA- los procesos comunicativos que se desarrollan en esta universidad redundan en una mayor facilidad y agilidad de los intercambios informativos. Al mismo tiempo, la activación de estos dispositivos parece propiciar la creación de espacios de trabajo colaborativos efectivos, supliendo algunas de las eventuales deficiencias asociadas a los sistemas educativos a distancia, deficiencias que habitualmente conducen a la deserción o el abandono por parte de los estudiantes.

Tal y como se evidencia en esta aproximación, Internet se erige en una herramienta 
favoreciendo la interiorización de los contenidos curriculares e introduciendo importantes mejoras en el seguimiento del proceso formativo que lleva a cabo alumno con el apoyo del profesor. El avance y el perfeccionamiento de las estrategias y de las herramientas comunicativas promete ayudar a implementar en el futuro vías alternativas para el desarrollo de la acción docente y parece anunciarnos que la impronta tecnológica constituirá una llave esencial para facilitar la adquisición de las habilidades competenciales que se exigen en el proceso formativo a los periodistas de la era digital.

\section{REFERENCIAS}

ANECA. (2005). Libro Blanco. Títulos de Grado en Comunicación. Recuperado el 6 de diciembre de 2011, de http://www.aneca.es/var/media/150336/libroblanco_comunicacion_def.pdf

Caballero Merino, A. I.; Fernández RAMOS, M. Y.; Pérez López, M. del C. \& Rodríguez Escanciano, I. (2008). El nuevo perfil del profesor universitario en el EEES: claves para la renovación metodológica. Valladolid: Servicio de Publicaciones.

Cardona Ossa, G. (2002). Tendencias Educativas para el siglo XXI. Educación Virtual, Online y @Learning. Elementos de discusión Edutec. Revista Electrónica deTecnología Educativa, 15. Recuperado el 6 de diciembre de 2011, de http://edutec.rediris.es/Revelec2/revelec15/cardona.pdf

Carrió Invernizzi, D.; Fernández Castrillo, C. \& Hidalgo Giralt, C. (2011). Sociallearning a través de Elluminate en UDIMA: tres experiencias en los grados de Periodismo, Historia y Turismo. Relada, 5. Recuperado el 6 de diciembre de 2011, de http://moodle.upm.es/adamadrid/file.php/1/web_VI_jornadas_ADA/comunicaci ones/30_Carrio.pdf

Friesen, N. (2009). Re-thinking e-learning research: foundations, methods and practices. Nueva Yok: Peter Lang.

Landeta Etxeberria, A. (2007). Buenas prácticas de e-learning y actividades didácticas innovadoras. San Sebastián: Asociación Nacional de Centros de E-Learning y Distancia.

Landeta Etxeberria, A. (2010). Nuevas tendencias de e-learning y Actividades didácticas innovadoras. Madrid: Ediciones Centro de Estudios Financieros. 
http://moodle.org/stats/

Prat Sedeño, J.(2006). Herramientas y estrategias de aprendizaje en línea para la formación de posgrado en gestión de la información digital en los medios de comunicación. Scire: representación y organización del conocimiento, 12(2) Recuperado el 6 de diciembre de 2011, de http://ibersid.eu/ojs/index.php/scire/article/viewArticle/1696

Real, E. (2005). Algunos interrogantes en torno a los estudios de Periodismo ante el nuevo Espacio Europeo de Educación Superior. Cuadernos de Información y Comunicación, 10: 268.

Santín Durán, M. (2009). La enseñanza on-line en el ámbito de la comunicación en España. Recuperado el 6 de diciembre de 2011, de

http:/ / www.dialogosfelafacs.net/descargas/APP_EJE2_Espana \%20\%20Marina \%20 Santin.pdf

Sicilia Urban, M. 1 Á. \& García Barriocanal, E. (2009). Aprendizaje y tecnologías de la información y la comunicación. Madrid: Ediciones Centro de Estudios Financieros.

Sierra, Javier \& Cabezuelo, Francisco (2009). Postperiodismo y formación en competencias digitales bajo el paraguas de Bolonia. Trabajo presentado al I Congreso Internacional de la Sociedad Latina de Comunicación. Tenerife. Universidad de La Laguna.

Zapata Ros, M. (2003). Redes telemáticas: educación a distancia y educación cooperativa. Revista Pixel_BIT. Revista de Medios y Educación, 20. Recuperado el 6 de diciembre de 2011, de

http://www.sav.us.es/pixelbit/pixelbit/articulos/n8/n8art/art83.htm

\section{Celia Berná Sicilia}

Doctora Europea en Filología Hispánica por la Universidad de Murcia y Licenciada en Periodismo por la Universidad de Murcia. Sus ámbitos de interés investigador se centran, además de en el estudio de las interacciones entre léxico, sintaxis y semántica, en el análisis de los factores que inciden en la comunicación en distintos tipos de discurso. Ha desarrollado su actividad docente e investigadora en distintos centros universitarios nacionales e internacionales, como la Universidad de La Sorbona (París IV), la Universidad de Zúrich (Suiza), la Universidad de Leipzig (Alemania) y la Universidad a Distancia de Madrid (UDIMA). Actualmente, es profesora en la Universidad Católica San Antonio de Murcia en las titulaciones de Comunicación y 


\section{Carolina Fernández Castrillo}

Doctora Europea en Ciencias de la Información por la Universidad Complutense de Madrid (Premio Extraordinario de Doctorado, UCM) y Doctora en Ciencias de la Comunicación por la Università degli Studi di Roma "La Sapienza". Su línea de investigación se centra en el estudio de las interrelaciones entre comunicación y cultura digital. Ha desarrollado su actividad docente e investigadora en diversos centros internacionales: Yale University (EE.UU), ZKM|Zentrum für Kunst und Medientechnologie Karlsruhe (Alemania), Universiteit Utrecht (Holanda), entre otros. En la actualidad es Profesora Adjunta del Grado de Periodismo y Directora del Máster en Educación y Nuevas Tecnologías de la Universidad a Distancia de Madrid (UDIMA). 